\title{
MIXED MEDULLARY AND PAPILLARY CARCINOMA OF THYROID - A RARE BUT DISTINCT CLINICAL ENTITY
}

\author{
Sidra Jahangir, Sajid Mushtaq, Asif Loya \\ Department of Pathology, Shaukat Khanum Memorial Cancer Hospital and Research Centre, Pakistan \\ Received: 12 August 2015 / Accepted: 15 September 2015
}

Mixed medullary and papillary thyroid carcinoma is a rare clinical entity that should be considered in the differential diagnosis of thyroid nodules showing variable histological patterns, particularly in patients with a family history of thyroid malignancy. Around 40 cases of mixed medullary and papillary carcinoma of thyroid have been reported in literature. ${ }^{[1]}$

The WHO classification of thyroid tumours includes an entity called mixed medullary and follicular cell carcinoma which describes tumours showing morphological features of both, medullary carcinoma with immunoreactivity for calcitonin and follicular (or papillary) carcinoma with immunoreactivity for thyroglobulin. The medullary and papillary components can be seen as separate components or merge unperceptively within the same tumour. ${ }^{[2]}$

According to the WHO, prognosis of mixed medullary and papillary carcinoma depends on medullary component because medullary carcinoma is usually the predominant component of this tumour. It is important that mixed papillary and medullary carcinoma not be confused with papillary pattern of medullary carcinoma. To differentiate between the two, expression of calcitonin and CEA in medullary carcinoma and thyroglobulin in papillary component along with nuclear features is helpful. ${ }^{[3]}$

We received a thyroidectomy specimen in a 50-year-old male, with a history of thyroid nodule. Specimen was received in multiple pieces.

Grossly multiple grey white firm nodules were identified within the thyroid. Two small lymph nodes were also present. Microscopically, two separate and distinct tumours were identified. One tumour exhibited papillary pattern with classic nuclear features including powdery chromatin, nuclear grooves and intranuclear inclusions. The second

Correspondence: Dr. Sidra Jahangir, Department of Pathology, Shaukat Khanum Memorial Cancer Hospital and Research Centre, Pakistan.

Email: jahangir_sidra@yahoo.com

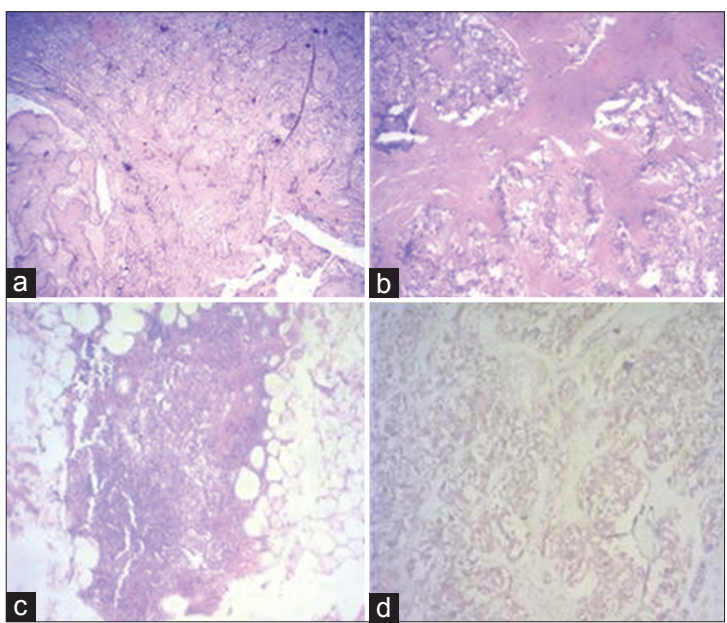

Figure 1: (a) Classical papillary carcinoma, original $\mathrm{H}$ and E section $\times 10$ view. (b) Medullary carcinoma, original $\mathrm{H}$ and E section $\times 20$ view. (c) Metastatic medullary carcinoma in lymph node, original $\mathrm{H}$ and $\mathrm{E}$ section $\times 20$ view. (d) Calcitonin expression in medullary carcinoma

showing solid nests with cells having hyperchormatic nuclei and salt and pepper chromatic pattern, and these cells were separated by homogenous eosinophilic material. Calcitonin was strongly positive in the second component confirming medullary carcinoma. Submitted lymph node was also positive for metastatic medullary carcinoma [Figure 1].

\section{Conflict of Interest}

The authors declare that they have no conflict of interest.

\section{References}

1. Hasney CP, Amedee RG. Mixed medullary-papillary carcinoma of the thyroid: A case report. Laryngoscope 2010;120 Suppl 4:S153.

2. Matias-Guiu X, Caixas A, Costa I, Cabezas R, Prat J. Compound medullary-papillary carcinoma of the thyroid: True mixed versus collision tumour [corrected]. Histopathology 1994;25:183-5.

3. Shukla S, Awasthi NP, Husain N. Papillary variant of medullary carcinoma thyroid. Indian J Pathol Microbiol 2014;57:151-2. 